\title{
Myelodysplastic syndrome without ring sideroblasts and with Janus kinase 2 gene mutation: An unusual case report
}

\author{
MARIA HELENA ORNELLAS ${ }^{1,2}$, MONIQUE DE FRANÇA SILVA ${ }^{1}$, \\ CRISTIANA SOLZA ${ }^{3}$, STELLA BEATRIZ SAMPAIO DE LUCENA GONÇALVES ${ }^{3}$, \\ LILIANE SILVA DE ALMEIDA ${ }^{1,2}$, JACKLINE DE PAULA AYRES-SILVA ${ }^{4}$, TAÍS LEITE SEIXAS ${ }^{5}$,

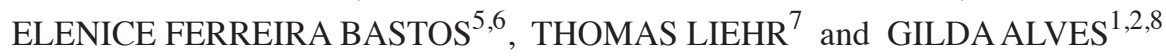 \\ ${ }^{1}$ Department of Pathology, Laboratory of Circulating Markers; ${ }^{2}$ Graduation Course in Medical Sciences, \\ Faculty of Medical Sciences (FCM), Rio de Janeiro 20550-170; ${ }^{3}$ Department of Haematology, \\ Pedro Ernesto University Hospital (HUPE), Rio de Janeiro State University (UERJ), Rio de Janeiro 20551-120; \\ ${ }^{4}$ Laboratory of Pathology, Oswaldo Cruz Foundation (FIOCRUZ), Rio de Janeiro 21040-360; ${ }^{5}$ Cytogenetics Laboratory, \\ Faculty of Medical Sciences, Rio de Janeiro State University (UERJ), Rio de Janeiro 20551-120; \\ ${ }^{6}$ Department of Medical Genetic, Fernandes Figueira Institute, Oswaldo Cruz Foundation (FIOCRUZ), \\ Rio de Janeiro 22250-020, Brazil; ${ }^{7}$ Jena University Hospital, Friedrich Schiller University, \\ Institute of Human Genetics, D-07743 Jena, Germany; ${ }^{8}$ Research Coordination, \\ National Cancer Institute (INCA), Ministry of Health, Rio de Janeiro 20230-130, Brazil
}

Received March 4, 2016; Accepted May 25, 2016

DOI: $10.3892 / \mathrm{mco} .2016 .947$

\begin{abstract}
Myelodysplastic syndrome (MDS) cases comprise a heterogeneous group of hematological disorders that are characterized by impaired hematopoiesis, with cytopenias of different grades and risk of developing acute myeloid leukemia. MDS may rarely be associated with thrombocytosis. In such cases, myelodysplasia and myeloproliferative disorders may overlap, making correct diagnosis difficult. We herein describe a case of MDS with thrombocytosis, Janus kinase 2 gene mutation-positive and Perls' staining-negative, which was initially classified as essential thrombocythemia (ET). This case highlights that MDS may be misdiagnosed as ET and inappropriate treatment may be initiated. Therefore, it is crucial to carefully combine all available data on morphology and immunophenotyping, and to perform the necessary molecular, cytogenetic and molecular cytogenetic analyses, in order to correctly diagnose this disease.
\end{abstract}

Correspondence to: Professor Gilda Alves, Department of Pathology, Laboratory of Circulating Markers, Faculty of Medical Sciences (FCM), Professor Manuel de Abreu Avenue, Vila Isabel, Rio de Janeiro 20550-170, Brazil

E-mail: galvesbrown@gmail.com

Key words: myelodysplastic syndrome, essential thrombocythemia, Janus kinase 2 gene, complex karyotype, aging

\section{Introduction}

Myelodysplastic syndromes (MDS) comprise a heterogeneous group of hematological disorders that are characterized by impaired hematopoiesis, resulting in cytopenias of different grades (1). Examination of the bone marrow (aspirate, biopsy, cytogenetics and flow cytometry) and the peripheral blood should reveal the morphological features of the disease and exclude from other conditions (2).

The World Health Organization (WHO) classifies MDS as refractory cytopenias with unilineage dysplasia, refractory anemia with ring sideroblasts, refractory anemia with excess blasts-1, refractory anemia with excess blasts-2, myelodysplastic syndrome-unclassified and myelodysplastic syndrome associated with isolated del 5q (3). In addition, the WHO classification recognizes a group of myeloid neoplasms with overlapping features of myelodysplastic syndromes (MDS) and myeloproliferative neoplasms (MPN) and places them under a separate category of MDS/MPN. Within this category, refractory anemia with ring sideroblasts with marked thrombocytosis (RARS) and MDS/MPN, unclassifiable (MDS/MPN-U) are also included (4).

The incidence of MDS is $\sim 3.8$ cases/100,000 habitants each year. It is rare in people $<40$ years old $(0.14 / 100,000$ habitants) and it increases according to aging (5). With regards to Brazil, the epidemiological data are restricted. In a retrospective study of $315 \mathrm{MDS}$ patients, an incidence of $7 \%$ was reported. They were classified as RARS, RA, RAEB and MDS/MPD-U (6).

Accurate prediction of a patient prognosis is useful to define the risk posed by the disease and the treatment options that are most appropriate. Since its publication in 1997, the International Prognostic Scoring System (IPSS) has been the tool used to stratify risk in these patients (7). In 2012, the 


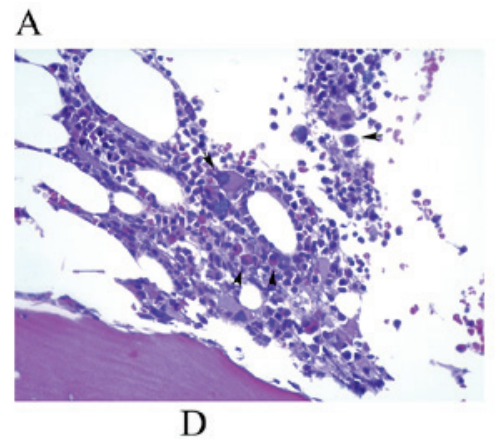

B

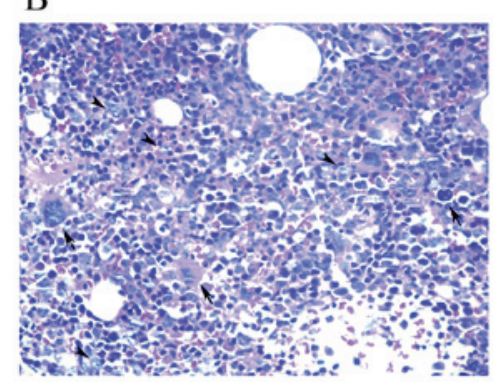

$\mathrm{E}$
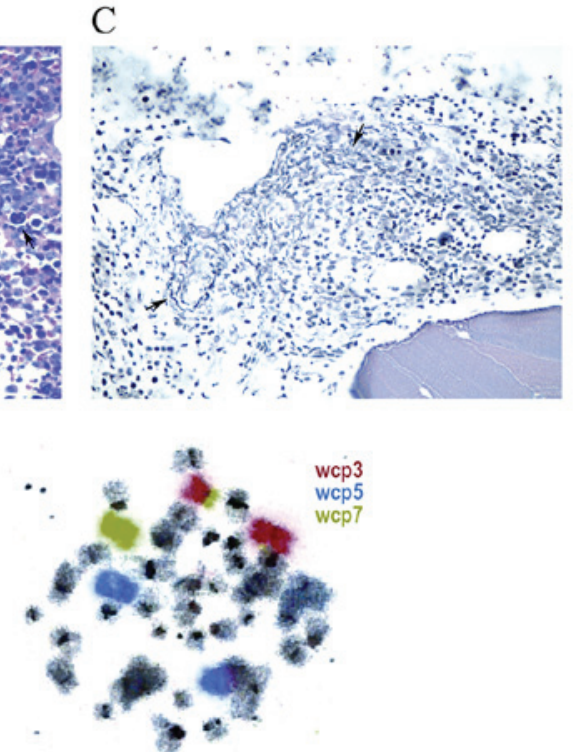

Figure 1. Pathology and cytogenetics findings. (A) Bone marrow biopsy at diagnosis [hematoxylin and eosin (H\&E) staining; magnification, $\mathrm{x} 400$ ] showing fragmented bone marrow represented by three intertrabecular spaces, displaying $\sim 60-70 \%$ overall cellularity. Three hematopoietic lineages were present. The granulocyte:erythroid ratio was 5:1. The granulocytic series displayed mature form however, there were common elements with left maturation. The normoblastic erythroid series were found in a small proportion (arrowheads). The megakaryocytes were increased in number, with moderate dysmorphism (small and hypolobulated; arrows). (B) Bone marrow biopsy in evolution (H\&E staining; magnification, x400). Bone marrow exhibiting 80\% overall cellularity. Three hematopoietic series were present. The granulocyte: Erythroid ratio was 5:1. The granulocytic series at various maturation stages exhibited blasts (arrowheads) in the center of the medullary cavity, corresponding to $\sim 15 \%$, with atypical localization of immature precursors. Megakaryocytes were present at an increased proportion, exhibiting dysmorphism, often in the form of small and hypolobulated cells and occasional microcytic forms, usually located in the center of the medullary cavity, predominantly isolated (arrows). Ferric pigment deposits were also present. (C) Bone marrow biopsy in evolution (reticulin staining; magnification, x400). A network of reticulin with numerous intersections was observed, particularly in the perivascular areas (arrows), defined as grade 1 myelofibrosis. (D) Trypsin-Giemsa banding. (E) Chromosome painting.

Revised International Prognostic Scoring System (IPSS-R) included the same major categories as the IPSS, but with significant improvements (7-9).

The treatment varies according to the level of risk. For low-risk patients the treatment consists of cytopenia correction. For anemia, the treatment consists of transfusion and erythropoietin (10). Lenalidomide was useful for patients with 5q-syndrome (11-13). Granulocyte-colony stimulating factor improves the neutropenia $(14,15)$. The hypomethylating agents, decitabine and azacitidine, can be used in low-risk patients who are transfusion dependent, and who have failed or are not candidates for the use of lenalidomide or growth factors $(14,15)$. In patients with high- and intermediate risk 2 , the treatment focuses on changing the natural history of the disease, due to the high risk of progression to acute myeloid leukemia (AML) and reduced survival. Therapeutic options include hypomethylating, targeted chemotherapy regimens for AML and allogeneic stem cell transplantation, which is the only curative treatment option. Allogeneic transplantation is reserved for patients $<65$ and human leukocyte antigen (HLA)-matched donor (1,5,13).

Thrombocytosis may be observed in certain MDS cases, mainly in cases of refractory anemia with ringed sideroblasts. However, in cases with the absence of ring sideroblasts, the MDS diagnosis is challenging and it may confound with myeloproliferative disorders (MPD), mainly if there is a Janus kinase 2 gene (JAK2) V617F mutation (16). This abnormality is much more common in this group of disorders compared to MDS. The present study describes an uncommon case of MDS that was initially classified as essential thrombocytosis (ET).

\section{Case report}

A 78-year-old woman presented with anemia and thrombocytosis for the last 6 months. The patient did not experience paresthesias, weight loss, pruritus, fever, headaches or arthralgia, and had not received transfusions. The initial laboratory tests revealed macrocytic anemia (mean corpuscular volume $=102 \mathrm{fl}$ ), with a hemoglobin concentration of $10.8 \mathrm{~g} / \mathrm{dl}$, a leukocyte count of $6.5 \times 10^{9} / 1$ without detected circulating blasts, and a platelet count of $812 \times 10^{9} / 1$. The renal and hepatic functions were within normal limits and lactate dehydrogenase (LDH) was found to be elevated to 419 IU/1 (normal range is $\leq 250 \mathrm{IU} / \mathrm{l}$ ). The ferritin and vitamin $\mathrm{B} 12$ levels were $167 \mathrm{ng} / \mathrm{ml}$ and $686 \mathrm{pg} / \mathrm{ml}$, respectively (within the normal range). Ultrasonography revealed mild splenomegaly $(12.8 \mathrm{~cm}$; normal size is $\leq 12 \mathrm{~cm}$ ) and portal vein thrombosis. Bone marrow aspiration revealed megakaryocytic hyperplasia and dysmegakaryopoiesis, without alterations of the erythroid or granulocytic lineages. A bone marrow biopsy revealed $70 \%$ hematopoietic cellularity with trilineage hematopoiesis, a normal ratio of myeloid to erythroid cells, megakaryocytic hyperplasia, dysmegakaryopoiesis, and no deposition of reticulin fibers (Fig. 1A). A cytogenetic investigation was not performed at this stage however, molecular analysis performed on a peripheral blood sample detected a $J A K 2$ mutation (V617F). The diagnosis was ET, according to the criteria proposed by the Polycythemia Vera Group (17). Therapy with hydroxyurea and aspirin were initiated. Two years later, the patient presented with 
weakness, fatigue, epistaxis and progressive dyspnea. Physical examination revealed tachycardia, tachypnea, fever and splenomegaly. The laboratory tests revealed normocytic anemia with a hemoglobin concentration of $6.0 \mathrm{~g} / \mathrm{dl}$, as well as thrombocytopenia $\left(61 \times 10^{9} / 1\right)$ and leukocytosis $\left(12 \times 10^{9} / 1\right)$ with circulating blasts. The therapy with hydroxyurea was interrupted and the patient received therapeutic blood transfusions. The renal and hepatic functions were normal, but $\mathrm{LDH}$ was elevated to 2,080 IU/1. The examination of bone marrow morphology revealed myelodysplasia: As shown in Fig. 1B, a bone marrow biopsy revealed that the marrow was markedly hypercellular $(85 \%)$ with atypical localization of immature precursors (ALIP), megakaryocytic hyperplasia with dysmegakaryopoiesis and grade 1 fibrosis (Fig. 1C). Immunophenotypic study identified dysplasia of the granulocytic and monocytic lineages, an increase in blasts positive for CD34, CD33 and CD117, and monocytosis, with aberrant expression of CD56, anti-HLA DR (dim) and partial expression of CD15. Perls' staining was performed and was negative for ring sideroblasts (results not shown). Banding cytogenetics analysis of the bone marrow revealed an aberrant karyotype (Fig. 1D), which was further characterized by molecular cytogenetics (Fig. 1E) using whole-chromosome painting probes for chromosomes 3,5 and 7 as follows: 45, XX, der(3) t(3;7) (p12;p13), del(5)(q12q33), -7, del(12) (p12)[18]/46, XX[2]. Tazocin treatment was initiated, but was interrupted on day 9 , due to the patient's progressively altered mental status, as well as development of a pulmonary infection. Soon after, the patient succumbed to septic shock.

\section{Discussion}

After the patient's death, the case was reclassified as MDS based on a comprehensive histopathological, immunophenotypic, molecular and cytogenetics review. At diagnosis, the patient presented with mild anemia and increased platelet count, and the results of the ultrasound examination were compatible with thrombosis. These characteristics may be common between ET and MDS, which may be misleading (18-20). The spleen size was within normal limits on ultrasonography. The World Health Organization characterized certain disorders as myelodysplastic/myeloproliferative neoplasms (MDS/MPN) in $2008(5,19)$. This group includes clonal myeloid diseases that exhibit an overlap between MDS and MPN. At the time of diagnosis, these patients exhibit clinical, laboratory, or morphological characteristics supporting the diagnosis of these two groups. Chronic myelomonocytic leukemia, juvenile myelomonocytic leukemia, atypical BCR-ABL1-negative chronic myeloid leukemia, MDS/MPN unclassifiable, refractory anemia with ring sideroblasts and thrombocytosis (RARS-T) are included (19). The patient in the present case was carrier of a JAK2 (V617F) mutation, which is more common in ET compared with MDS patients $(3,16,19-22)$. The $J A K 2$ (V617F) mutation is described in $>95 \%$ of polycythemia vera and $50-60 \%$ of ET and myelofibrosis patients. However, in MDS, the JAK2 (V617F) mutation is found mainly in patients with RARS-T (refractory anemia with ring sideroblasts), which was excluded by Perls' staining (23-25). The histopathological and immunophenotypic studies as well as the presence of micromegakaryocytes and ALIP favored the diagnosis of MDS. In ET the presence of micromegakaryocytes are uncommon (Fig. 1C). Immunophenotypic studies also support this hypothesis, as described in the present study. Cytogenetics was also valuable for the diagnosis in this case. Chromosome banding combined with molecular cytogenetics was useful and indicative of the presence of a myeloid disorder. The presence of chromosomal abnormalities, such as abnormalities of $3 q$ or monosomy 7 , is a known indicator of an adverse clinical outcome (9). The classical risk factor associated with MDS is ageing. However, to learn more about this case, the son of the patient consented to being interviewed after her death: He reported that the deceased patient and her family had no previous history of malignant disease. The patient was a non-smoker and did not regularly consume alcoholic beverage. She was a housewife, but had lived in close proximity to a leather factory for 35 years, which may have contributed to the onset of her disease, since carcinogenic chemical substances are associated with this type of industry $(24,25)$.

In conclusion, due to its heterogeneity, MDS may be misdiagnosed as ET and inappropriate treatment may be initiated. Thus, it is crucial to carefully combine all available data on morphology and immunophenotype, and to conduct the necessary molecular, cytogenetic and molecular cytogenetic analyses, in order to reach the correct diagnosis and administer the optimal treatment.

The present study was approved by the Ethical Board of the Pedro Ernesto University Hospital (HUPE); registration number, 0010.0.228.000-07. The patient's son signed an informed consent regarding the publication of the case details.

\section{References}

1. Garcia-Manero G: Myelodysplastic syndromes: 2015 Update on diagnosis, risk-stratification and management. Am J Hematol 90: 831-841, 2015.

2. Nimer SD: Myelodysplastic syndromes. Blood 111: 4841-4851, 2008.

3. Brunning RD, Orazi A, Germing U, Le Beau Porwit, Baumann I, Vardiman JW and Hellstrom-Lindberg E: Myelodysplastic syndromes/neoplasms, overview. In: WHO Classification of Tumours of Haematopoietic and Lymphoid Tissues. Swerdlow SH, Campo E, Harris NL, Jaffe ES, Pileri SA, Stein H, Thiele J and Vardiman JW (eds). 4th edition. IARC Press, Lyon, pp87-93, 2008.

4. Foucar K: Myelodysplastic/myeloproliferative neoplasms. Am J Clin Pathol 132: 281-289, 2009.

5. Sekeres MA: The epidemiology of myelodysplastic syndromes. Hematol Oncol Clin North Am 24: 287-294, 2010.

6. Cabello AI, Collado R, Ruiz MA, Martínez J, Navarro I, Ferrer R, Sosa AM and Carbonell F: A retrospective analysis of myelodysplastic syndromes with thrombocytosis: Reclassification of the cases by WHO proposals. Leuk Res 29: 365-370, 2005.

7. Greenberg P, Cox C, LeBeau MM, Fenaux P, Morel P, Sanz G Sanz M, Vallespi T, Hamblin T, Oscier D, et al: International prognostic scoring system for evaluating prognosis in myelodysplastic syndromes. Blood 89: 2079-2088, 1997.

8. Greenberg PL, Tuechler H, Schanz J, Sanz G, Garcia-Manero G, Solé F, Bennett JM, Bowen D, Fenaux P, Dreyfus F, et al: Revised international prognosis scoring system for myelodysplastic syndromes. Blood 120: 2454-2465, 2012.

9. Haase D, Germing U, Schanz J, Pfeilstöcker M, Nösslinger T, Hildebrandt B, Kundgen A, Lübbert M, Kunzmann R, Giagounidis AA, et al: New insights into the prognostic impact of the karyotype in MDS and correlation with subtypes: Evidence from a core dataset of 2124 patients. Blood 110: 4385-4395, 2007.

10. Moyo V, Lefebvre P, Duh MS, Yektashenas B and Mundle S: Erythropoiesis-stimulating agents in the treatment of anemia in myelodysplastic syndromes: A meta-analysis. Ann Hematol 87: 527-536, 2008. 
11. List A, Dewald G, Bennett J, Giagounidis A, Raza A, Feldman E, Powell B, Greenberg P, Thomas D, Stone R, et al: Lenalidomide in the myelodysplastic syndrome with chromosome $5 \mathrm{q}$ deletion. N Engl J Med 355: 1456-1465, 2006.

12. Fenaux P, Giagounidis A, Selleslag D, Beyne-Rauzy O, Mufti G, Mittelman M, Muus P, Te Boekhorst P, Sanz G, Del Cañizo C, et al: A randomized phase 3 study of lenalidomide versus placebo in RBC transfusion-dependent patients with low-/intermediate-1 risk myelodysplastic syndromes with del5q Blood 118: 3765-3776, 2011.

13. Fenaux P and Adès L: How we treat lower-risk myelodysplastc syndromes. Blood 121: 4280-4286, 2013.

14. Fenaux P, Haase D, Sanz GF, Santini V and Buske ESMO Guidelines Working Group: Myelodysplastic syndromes: ESMO clinical practice guidelines for diagnosis, treatment and follow-up. Ann Oncol 25 (Suppl 3): iii57-iii69, 2014.

15. Garcia-Manero G: Myelodysplastic syndromes: 2014 update on diagnosis, risk-stratification, and management. Am J Hematol 89 : 97-108, 2014

16. Jeromin S, Haferlach T, Weissmann S, Meggendorfer M, Eder C, Nadarajah N, Alpermann T, Kohlmann A, Kern W, Haferlach C and Schnittger S: Refractory anemia with ring sideroblasts and marked thrombocytosis cases harbor mutations in SF3B1 or other spliceosome genes accompanied by JAK2V617F and ASXL1 mutations. Haematologica 100: e125-e127, 2015.

17. Murphy S, Peterson P, Iland $\mathrm{H}$ and Laszlo J: Experience of the Polycythemia Vera study group with essential thrombocythemia: A final report on diagnostic criteria, survival, and leukemic transition by treatment. Semin Hematol 34: 29-39, 1997.

18. Steensma DP: JAK2 V617F in myeloid disorders: Molecular diagnostic techniques and their clinical utility: A paper from the 2005 William Beaumont Hospital Symposium on Molecular Pathology. J Mol Diagn 8: 397-411, 2006.
19. Vardiman JBR, Bennett JM, Bain BJ, Baumann I, Thiele J and Orazi A: Myelodysplastic/myeloproliferative neoplasm, unclassifiable. In: WHO Classification of Tumours of Haematopoietic and Lymphoid Tissues. Swerdlow SH, Campo E, Harris NL, Jaffe ES, Pileri SA, Stein H, Thiele J and Vardiman JW (eds.) IARC Press, Lyon, pp85-86, 2008.

20. Orazi A and Germing U: The myelodysplastic/myeloproliferative neoplasms: Myeloproliferative diseases with dysplastic features. Leukemia 22: 1308-1319, 2008.

21. Szpurka H, Tiu R, Murugesan G, Aboudola S, Hsi ED, Theil KS Sekeres MA and Maciejewski JP: Refractory anemia with ringed sideroblasts associated with marked thrombocytosis (RARS-T), another myeloproliferative condition characterized by JAK2 V617F mutation. Blood 108: 2173-2181, 2006.

22. Malcovati L and Cazzola M: Refractory anemia with ring sideroblasts. Best Pract Res Clin Haematol 26: 377-385, 2013.

23. Patnaik MM and Tefferi A: Refractory anemia with ring sideroblasts and RARS with thrombocytosis. Am J Hematol 90: 549-559, 2015.

24. Mikoczy Z, Schütz A and Hagmar L: Cancer incidence and mortality among Swedish leather tanners. Occup Environ Med 51: 530-535, 1994.

25. Mikoczy Z, Schütz A, Strömberg U and Hagmar L: Cancer incidence and specific occupational exposures in the Swedish leather tanning industry: A cohort based case-control study. Occup Environ Med 53: 463-467, 1996. 